\title{
History of posterior thoracic instrumentation
}

\author{
HarshPal Singh, B.S., Scott Y. Rahimi, M.D., DAVID J. YeH, M.D., \\ AND DAVID FLOYD, M.D. \\ Department of Neurosurgery, Medical College of Georgia, Augusta, Georgia
}

\begin{abstract}
The term "backbone" appears in many expressions used in modern day society. In any scenario, it has one central meaning: stability. Best defined as a foundation that is able to sustain multiple stressors without adversely affecting integrity, the commonly and appropriately termed backbone of humans is the spinal column. As the central focus of stability in our species, the spine is subject to a great degree of trauma and mechanical forces. A variety of methods have been developed throughout history in the treatment of spinal column injury. Initial treatment involved the use of simple traction devices for the reduction of spinal fractures; these have evolved to include the current insertion of spinal instrumentation. The authors review the historical treatment and development of posterior instrumentation for thoracic spinal injury.
\end{abstract}

KEY WORDS • posterior instrumentation - thoracic spinal injury

\section{HISTORY OF THE TREATMENT OF THORACIC SPINAL INJURY}

Various modalities of treatment have evolved over centuries in the treatment of spinal column injury, with the earliest methods found in the Edwin Smith papyrus, which dates as far back as $1550 \mathrm{BC} .{ }^{5}$ Early therapies called for the prescription of rest and application of dressings to wounds. Unfortunately, a spinal injury remained a relatively morbid injury largely due to lack of knowledge about the anatomy of the spine. Spinal anatomy became better defined in the ancient Greek era. Although human dissection was prohibited in Greek society, knowledge was obtained through the observation of athletes in the gymnasium, the examination of cadavers in the battlefield, and the dissection of animals. ${ }^{6}$

The physician and philosopher Hippocrates (460-377 BC) was considered the father of spinal surgery. In his treatise On the Nature of Bones, Hippocrates provided a detailed description of the different segments of the spine. He described three levels of spinal vertebrae: those supraclavicular in location, those with costal articulations, and those located between the chest and pelvis. ${ }^{6}$ The first documented schematic for the treatment of nonparalytic spinal injury is found in the treatises of Hippocrates. ${ }^{6}$ Treatment consisted of fracture reduction followed by

Abbreviations used in this paper: $\mathrm{CT}=$ computerized tomography; VB = vertebral body. immobilization. A variety of devices were created to achieve traction and reduction; these included the Hippocratic ladder, the Hippocratic board, and the traction bed. Similar devices appeared in ancient Arabic and Chinese literature as well. Hippocrates speculated on the use of transabdominal repair for anterior spinal dislocation in his writings, although he stated that it could only be performed postmortem. ${ }^{6}$

Surgical intervention in a living patient was first proposed by Paulus of Aegina who lived in the seventh century. Paulus recommended that a fracture associated with paralysis should be treated by removal of bone fragments that cause a neurological deficit. It has been documented that, in addition to using traction devices, Paulus used a red hot iron during spinal interventions. ${ }^{5}$ In the 15 th century treatise Cerrahiyetul Haniye, the Turkish physician Serefeddin Sabuncuoglu also described traction methods and the use of cauterization in the treatment of spinal injury. ${ }^{8}$ His treatise contains illustrations of instruments needed for the surgical procedures as well as the traction devices that were used. In the 1700s Sir Percival Pott surgically opened and drained paravertebral abscesses caused by tuberculous spondylitis, ${ }^{5}$ a condition that now is referred to as Pott disease.

The historical treatment of thoracic spinal injury has been associated with numerous complications. Although these complications have not been comprehensively described in the literature, we can infer that deep vein thrombosis, prolonged disability, muscle atrophy, ligamentous stricture, and paralysis were among those complications. 


\section{EVOLUTION OF SURGICAL INTERVENTION}

The ultimate goal in surgical intervention for fixation of spinal injury is to achieve a nonpathological state between neural elements and their supporting soft tissues and osseous structures. ${ }^{12}$ Surgical intervention for spinal injury started out as a slowly evolving process; the predominant impediment to its use was infection due to the lack of sterile technique and absence of effective antibiosis. The work of Semmelweis and Lister provided a necessary adjunct to help prevent infection, thereby advancing the process of invasive intervention. A key event that led to an increase in the performance of all surgical procedures, including spinal intervention, was the incorporation of antisepsis into standard operating procedure in $1867 . .^{5}$

In 1886, MacEwen documented the first laminectomy. In the early 1900s Menard introduced the costotransversectomy, which was performed to relieve of pressure on the vertebral canal in paraplegic patients with tuberculous spondylitis. DeForest Willard soon repeated the same procedure for the same indication in the US. ${ }^{5}$ Using silver wire to facilitate fusion, Berthold Earnest Hadra accomplished dorsal stabilization of a cervical fracture-dislocation in 1891. Autologous tibial bone transplants were used to stabilize larger defects of VBs, which were found among patients afflicted with Pott disease. ${ }^{7}$ In 1909, Fritz Lang affixed rigid celluloid rods to either side of the spinous processes by using silk thread and steel wires ${ }^{7}$ to stabilize the spine. Lang discovered that internal fixation induced more timely healing than immobilization therapy alone. ${ }^{7}$ Nonetheless, these therapies were more pertinent to the cessation of progression of spinal deformity than to the correction of this disorder.

Imaging modalities aid in the diagnosis and treatment of spinal fractures. Radiographic imaging was first accomplished by Wilhelm Conrad Röntgen in 1895. Using this method spinal views were obtained only in an anteroposterior fashion until 1925, when Davis obtained the first usable lateral view. ${ }^{5}$ Radiographic imaging and newer imaging modalities such as CT scanning and magnetic resonance imaging have provided surgeons with a blueprint on which they can base their plan of invasive fixation.

\section{MODERN POSTERIOR THORACIC INSTRUMENTATION}

\section{Harrington Instrumentation System}

The treatment of patients suffering from progressive neuromuscular scoliosis fell to Dr. Paul Harrington of Houston, Texas. In 1953, Harrington began to develop what is now known as the Harrington rod system. The initial procedure involved the placement of facet screws to correct the position of facet joints. Initial postoperative results were favorable; however, a longer follow-up time demonstrated poor results. ${ }^{4}$ The addition of hooks and stainless steel rods to the construct were then implemented in an attempt to provide a stronger construct. Again the postoperative results seemed to be promising, but longterm follow-up studies demonstrated a recurrence of the deformity, loosening of the rods, and the development of flat-back syndrome in these patients. The failure of the spinal hardware led Harrington to work with engineers to develop metal instrumentation that could sustain a greater degree of repetitive stress. It also was decided to implement bone graft insertion simultaneously for the purposes of fusion and supplementation of fixation strength. ${ }^{7}$ The use of Harrington rods was initially indicated for the treatment of scoliotic spinal deformity. Later the indication for use expanded to include stabilization of traumatic injury, degenerative disease, and conditions brought about by tumor. ${ }^{3}$ In most cases, the use of a postoperative brace for a 6-month period was recommended.

The Harrington rod instrumentation system provides for a long rigid construct. Hence, its use disrupts the normal sagittal spinal contours found in the thoracolumbar spine. The system also fails to provide the necessary lordosis or rotational control at the thoracolumbar junction. ${ }^{3}$ Other complications of posterior Harrington rod instrumentation include hook dislodgment, hook-rod disengagement, and laminar fracture. Because flexion and rotation about the upper hook is not well controlled with the use of the Harrington construct, in addition to the reduced hook-lamina contact forces become concentrated on the laminar edge, resulting in hook dislodgment or laminar fracture. Laminar fracture can also result from osteoporosis, extensive laminotomy, or excessive distraction. Hook-rod disengagement occurs when one fails to leave a 1-cm length of rod beyond the proximal hook position; disengagement occurs during flexion. ${ }^{3}$ Although not restricted to the thoracic spine, dural compromise from insertion of laminar hooks can also be a complication. This is illustrated by Fig. 1, which shows dural compromise from an insertion of a laminar hook into the cervical spine.

Although fraught with complications, Harrington rod instrumentation provided a means of thoracic stabilization in afflicted patients. These patients were followed postoperatively and their outcomes were analyzed. The net result of these studies demonstrated that, independent of construct dexterity and strength, thoracic stabilization without

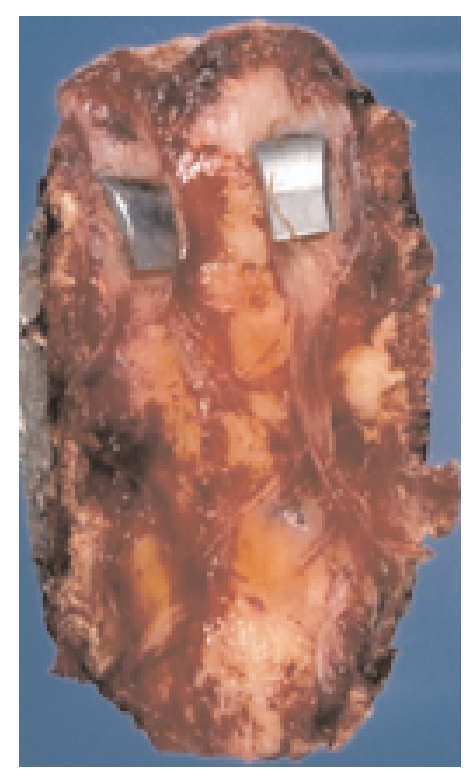

Fig. 1. Photograph showing dural compromise caused by the insertion of laminar hooks. 
fusion augmentation would inevitable result in hardware failure.

\section{Luque Instrumentation System}

In the wake of the work on spine stabilization done by Paul Harrington, Dr. Eduardo Luque, a noted physician in Mexico City, developed a similar rod system in 1976. The Luque rod system consisted of long, contoured cylindrical rods measuring $3 / 16$ or $1 / 4$ in in diameter. The rods were affixed to the spine by using 16- or 18-gauge sublaminar wires at multiple segments along its length. The contoured rods provided immediate stabilization of the thoracolumbar spine and the patients did not require postoperative braces in many cases. ${ }^{1}$ Dr. Luque worked with indigent patients who had to travel many miles to receive his care. Unfortunately, the use of postoperative braces and attendance at follow-up visits were difficult in this patient population.

Fusion augmentation was achieved with greater success along the multiple points of fixation in the Luque rod system compared with the Harrington system. Nevertheless, the use of sublaminar wires met with a greater degree of neurological complication. Dysesthesia was seen in as many as $10 \%$ of patients. ${ }^{3}$ Other neurological complications secondary to passage of sublaminar wires include cord ischemia from compromised blood flow and postoperative paraplegia. In cases of postoperative paraplegia, the patient should be immediately returned to the operating room for removal of the instrumentation for optimal outcomes. The sublaminar wires should be carefully extracted to prevent any additional neural trauma.

\section{Pedicle Screw Fixation}

The earlier systems involved the use of hooks and wires to accomplish stabilization. A newer and more rigid construct has been established by the use of pedicle screw-assisted instrumentation. Originally described by Michele and Krueger in $1949,{ }^{1}$ pedicle screw fixation has gained much popularity in recent time. Transpedicular fixation is achieved by the passage of a screw in a posteroanteromedial direction through the pedicle into the VB.
One of two trajectories is selected by the surgeon: the anatomical or the straight-forward approach ${ }^{11}$ (Fig. 2). The anatomical approach provides the largest possible bone channel for screw placement, but it requires the use of a multiaxial screw. The straight-forward approach ${ }^{14}$ is a slightly modified Roy-Camille approach that was popularized by Suk and Lenke. In this approach fixed head screws are used. The screws are then stabilized by linkage to a rod, plate, or internal fixator (Fig. 3).

Advantages in the use of pedicle screws include universal application, fixation strength, and a shorter construct length. ${ }^{3}$ Posterior elements do not need to be intact for placement of the screws; therefore, these screws can be used in cases of traumatic thoracic injury in which there are spinal pathological conditions or in cases in which there is no degeneration of the posterior element. In contrast to other instrumentation systems, pedicle screw fixation offers stabilization of all three mechanical columns of the spine. The biomechanical properties of the thoracic spine differ from those of the cervical and lumbar regions. The load-bearing axes of cervical and lumbar spinal segments reside in the dorsal aspect of the VB, whereas in the thoracic region the same load-bearing axis is located in the ventral aspect of the VB. ${ }^{12}$ Through biomechanical testing and the work of Roy-Camille, the pedicle has been determined to offer the site of the most rigid fixation. ${ }^{3}$ In addition, a shorter construct can be used with pedicle screws because the screw-bone interface has greater strength than a hook-bone or wire-bone interface; this helps limit the number of segments to be fused, thereby helping maintain the normal sagittal contour. ${ }^{3}$

The potential risk of damage to nervous and vascular structures is the major limitation associated with the insertion of thoracic pedicle screws. Medial intrusion of instrumentation into the spinal canal obviously risks injury to neural elements. The most desirable location of pedicle screws is intrapedicular, because this provides a higher maximum fusion construct stability when compared with extrapedicular (pedicle/rib) fixation. ${ }^{9}$ The thoracic pedicles are small and vary in their angle of direction compared with other spinal pedicles. A morphometric analysis performed in a series of cadaveric thoracic vertebrae dem-
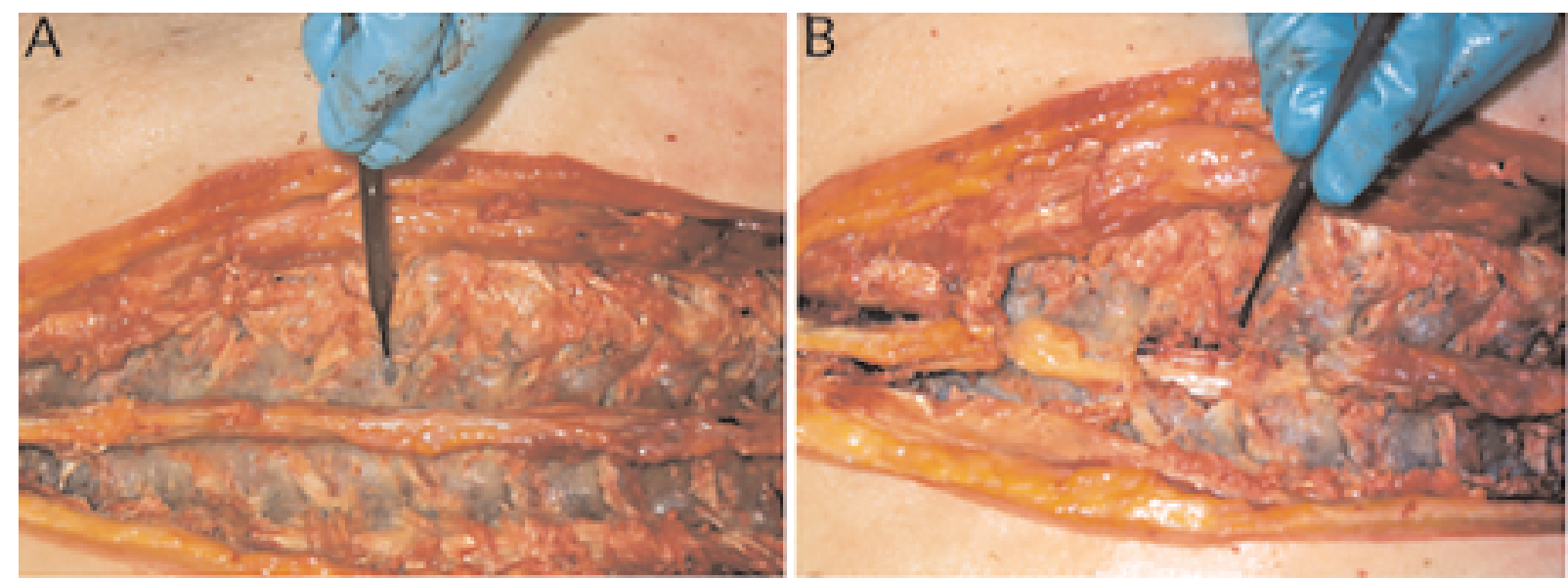

Fig. 2. Photographs obtained during a cadaveric dissection of the thoracic spine demonstrating the straight-forward (A) and anatomical (B) approaches to pedicle screw insertion. 


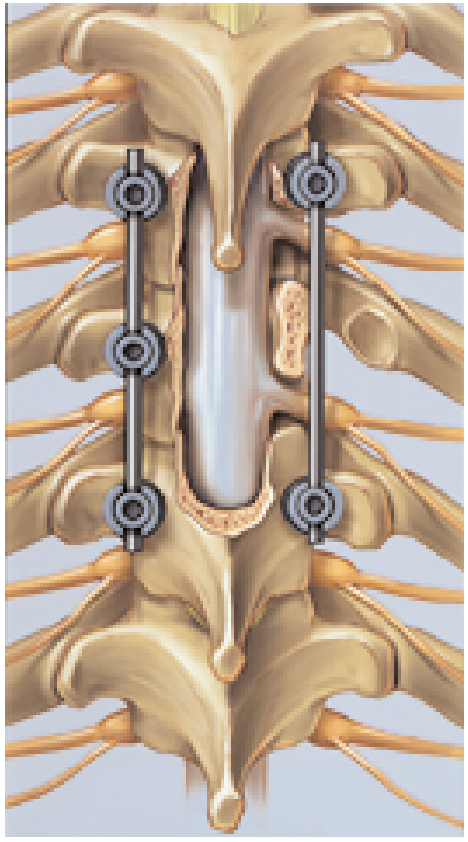

Fig. 3. Artist's drawing demonstrating a series of thoracic pedicle screws connected by rod placement.

onstrated that $35 \%$ of all pedicles were less than $5 \mathrm{~mm}$ wide, which is less than the smallest screw contained in a number of pedicle screw implants. ${ }^{2}$ Another cadaveric study has shown that conventional placement of thoracic pedicle screws resulted in an accuracy rate ranging from 45 to $85 \% .{ }^{11}$ When the accuracy of stereotactic thoracic pedicle screw placement was evaluated using CT scans, an $8.5 \%$ rate of cortical penetration was found and only $2.2 \%$ of all screws that were placed were thought to have produced structurally significant penetrations. ${ }^{15}$ Fluoroscope-assisted laser targeting has been shown to have a $99 \%$ rate of accuracy. ${ }^{13}$ Some surgeons have suggested that there is an inherent tendency to overestimate misplacement; CT scanning is the most valid tool to assess the accuracy of screw placement. ${ }^{10}$

Cinotti and colleagues ${ }^{2}$ have suggested that preoperative assessment of pedicle size should be performed if instrumentation is needed to treat levels between T-4 and T- 8 because the pedicles may not be suitable for instrumentation because of their small width. ${ }^{2}$

\section{CONCLUSIONS}

Treatment of thoracic spinal injury has evolved from simple traction methods and local wound care to invasive treatments involving placement of spinal hardware, as discussed earlier in this paper. This evolution of surgical intervention has been aided by the understanding of infective processes as well as by the development of medcations to treat infection. Imaging techniques have also helped us gain a better understanding of the patient's specific disease process in addition to aiding in the plan of care. Posterior stabilization of pathological conditions of the thoracic spine can be accomplished using different types of instrumentation, including Harrington rods, Luque rods, and pedicle screws. Of these systems, pedicle screw fixation provides the most stable construct for patients. The thoracic pedicle has an intricate anatomy that leads to a greater degree of difficulty in screw insertion. Image-guided placement of pedicle screws possibly provides a more safe and effective outcome.

\section{References}

1. Albert TJ, Jones AM, Balderston RA: Spinal instrumentation, in Rothman RH, Simeone FA (eds): The Spine, ed 3. Philadelphia: WB Saunders, 1992, Vol II, pp 1777-1796

2. Cinotti G, Gumina S, Ripani M, et al: Pedicle instrumentation in the thoracic spine. A morphometric and cadaveric study for placement of screws. Spine 24:114-119, 1999

3. Cotler JM, Simpson M, An HS: Principles, indications, and complications of spinal instrumentation: a summary chapter, in An HS, Cotler JM (eds): Spinal Instrumentation. Baltimore: Williams \& Wilkins, 1992, pp 435-453

4. Harrington PR: Treatment of scoliosis: correction and internal fixation by spine instrumentation. June 1962. J Bone Joint Surg Am 84-A:316, 2002

5. Knoeller SM, Seifred C: Historical perspective: history of spinal surgery. Spine 25:2838-2843, 2000

6. Marketos SG, Skiadas P: Hippocrates. The father of spine surgery. Spine 24:1381-1387, 1999

7. Mohan AL, Das K: History of surgery for the correction of spinal deformity. Neurosurg Focus 14 (1):Article 1, 2003

8. Naderi S, Acar F, Arda MN: History of spinal disorders and Cerrahiyetul Haniye (Imperial Surgery): a review of a Turkish treatise written by Serefeddin Sabuncuoglu in the 15th century. Historical vignette. J Neurosurg (Spine 3) 96:352-356, 2002

9. OBrien MF, Wood J, Lowe TG, et al: Thoracic Pedicle vs Pedicle/Rib Screw Fixation: A Biomechanical Study. (http://www.spineuniverse.com/displayarticle.php/article377.ht ml) [Accessed 15 December 2003]

10. Rao G, Brodke DS, Rondina M, et al: Comparison of computerized tomography and direct visualization in thoracic pedicle screw placement. J Neurosurg (Spine 2) 97:223-226, 2002

11. Rosner MK, Polly DW Jr, Kuklo TR, et al: Thoracic pedicle screw fixation for spinal deformity. Neurosurg Focus 14 (1): Article 7, 2003

12. Schlenk RP, Kowalski RJ, Benzel EC: Biomechanics of spinal deformity. Neurosurg Focus 14 (1):Article 2, 2003

13. Schwend RM, Dewire PJ, Kowalski TM: Accuracy of fluoroscopically assisted laser targeting of the cadaveric thoracic and lumbar spine to place transpedicular screws. J Spinal Disord 13:412-418, 2000

14. Suk SI, Lee CK, Kim WJ, et al: Segmental pedicle screw fixation in the treatment of thoracic idiopathic scoliosis. Spine 20: 1399-1405, 1995

15. Youkilis AS, Quint DJ, McGillicuddy JE, et al: Stereotactic navigation for placement of pedicle screws in the thoracic spine. Neurosurgery 48:771-779, 2001

Manuscript received November 17, 2003.

Accepted in final form December 11, 2003.

Address reprint requests to: Scott Y. Rahimi, M.D., Department of Neurosurgery, Medical College of Georgia, BI-3088, 1120 15th Street, Augusta, Georgia 30912. email: dyeh@mail.mcg.edu. 